\title{
DETERMINATION OF THE ELECTRIC FIELD VECTOR TWO DIMENSIONAL DISTRIBUTION INSIDE A HOLLOW CATHODE USING THE LASER STARK SPECTROSCOPY OF NaK
}

\author{
M.P. ALBERTA and J. DEROUARD \\ Laboratoire de Spectrométrie Physique and CELPHYRA (UA CNRS 08), Université Joseph Fourier, \\ Grenoble I, BP. 87, F-38402 St Martin d'Hères cedex, France
}

\begin{abstract}
.
We demonstrate the possibility to perform space and time resolved measurements of the electric field vector in axisymetric discharges using a laser Stark spectroscopy technique. The method is applied to map out the electric field in discharges through rare gases-potassium vapor mixtures. Such mixtures are chosen because they contain traces of NaK molecules, which is used as a sensitive probe of the electric field. Results are presented concerning the study of DC and transient hollow cathode discharges.
\end{abstract}

\section{INTRODUCTION.}

In glow discharges the electric field can be considered as the key parameter which determines the motion of the charged particles and the ionization processes. Its measurement is thus of primary importance for a better understanding of the mechanisms and the carrying out of realistic numerical models.

Recently several optical laser spectroscopic techniques $1-10$ have been developed to probe the electric field in discharges. Most of them rely on the observation of Stark shifts ${ }^{1-4}$ or mixings ${ }^{5-9}$ in atoms and molecules. However, most of these studies are restricted to unidimensional discharges, where the electric field vector is assumed to be parallel to a given axis, perpendicular to the electrodes and more or less uniform radially. Deviations from this simplest behavior are important to consider, with respect to the finite size of the electrodes and/or the vessel, the boundary potentials, the edge effects, the lateral spreading or stability of discharges etc...It is also very interesting to observe the time dependence of the field in non steady state discharges.

\section{EXPERIMENTAL.}

Our method is based on the dependence of the laser induced fluorescence spectrum of the NaK molecule on the electric field ${ }^{7}$ (Fig. 1) Because of Stark mixing effects, the 
electric field induces 5,7 the appearance of "forbidden" ("Q") lines in the fluorescence spectrum, whose intensity is borrowed from the intensity of the "allowed" (" $\mathrm{P}$ " or " $R$ ") lines. In addition, because the Stark mixing depends on the orientation of the molecules with respect to the direction of the elctric field vector $\mathrm{E}$, the Stark induced "forbidden" fluorescence is polarized along a direction related to that of $\mathrm{E}$.

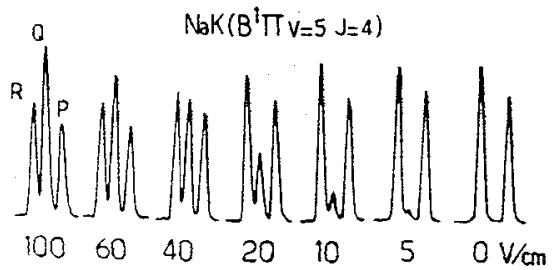

Fig. 1 Fluorescence spectrum of laser excited $\mathrm{NaK}$ for different values of the applied electric field. The $Q / R$ intensity ratio serves as a measure of the E-field magnitude, while the polarization of the $Q$ fluorescence yields the direction of the $E$ - field vector.

A sketch of the experimental set up is shown on Fig. 2.The probe laser beam is send into the discharge, perpendicularly to the axis of the electrodes. The laser induced fluorescence detected at right angle is spectrally resolved and polarization analyzed. The probed volume ( $1 \mathrm{~mm}^{3}$ or less) is determined by the crossing of the line of sight of the fluorescence detection with the laser beam. To map out the discharge, we move the discharge cell with respect to the optics. Time resolution ( $25 \mathrm{~ns}$ or less) is obtained from the analysis of the time dependence of the fluorescence signals ${ }^{8}$.

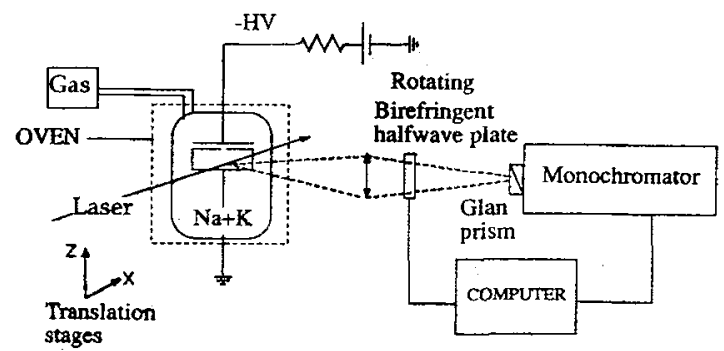

Fig. 2 Experimental set up.

\section{APPLICATION TO THE STUDY OF HOLLOW CATHODES DISCHARGES}

\subsection{Hollow Cathode discharges}

Hollow cathodes glow disharges are present in many applications. Especially interesting are "pseudosparks" devices, which are switches whose current rise characteristics (several tens of $\mathrm{kA}$ in some tens of nanoseconds, with a short time jitter) are very promising ${ }^{12-13}$. In the simplest configuration ( Fig.3) they consist of a planar anode separated from the parallel face of a cathode by a distance small enough 
such that a breakdown cannot occur between the two plates (Paschen curve). The cathode has a central hole, whose diameter is much less than the diameter of the electrode, which is open on a hollow backspace. Upon application of a voltage an ion space charge and hence a space charge field sufficiently high to increase the electron multiplication build up in the hollow cathode, and a plasma is formeci.

\subsection{Steady state regime}

Fig.3 shows an example of the electric field map observed in steady state conditions. Two sheaths are visible in the hollow cathode, between which the electrons are confined. This gives rise to a very efficient multiplication of charges ${ }^{13}$ and eventually to a fast current rise if the applied voltage were high enough. We observe also the distorsion of the field lines close to the hole, where it exists a very intense glow.

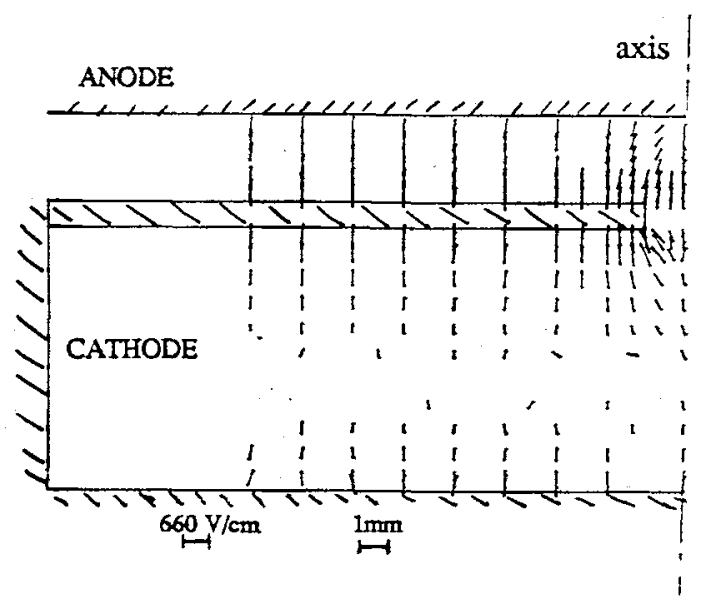

Fig. 3 Electric field map in a hollow cathode DC discharge. Conditions are: Gas: $\mathrm{Ar}+1.8 \% \mathrm{~K}\left(650 \mathrm{mT}, 220^{\circ} \mathrm{C}\right)$; Applied voltage: $116 \mathrm{Volt}, \mathrm{I}=8 \mathrm{~mA}$.

\subsection{Pulsed regime}

Fig 4 shows the building of the space charge in the volume of the hollow cathode as a function of time and position following the application of a transient hight voltage. The measurements have been made about $10 \mathrm{~mm}$ of the axis where the Electric field is roughly uniform radially. Note the appearence of the sheaths, the relatively long delay between the voltage and the current raise and the optical emission intensity, which shows two phases. 

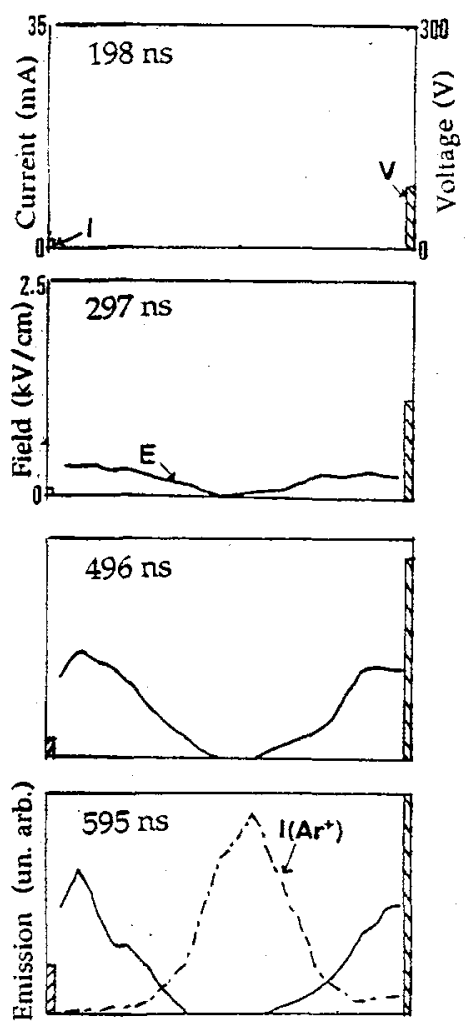
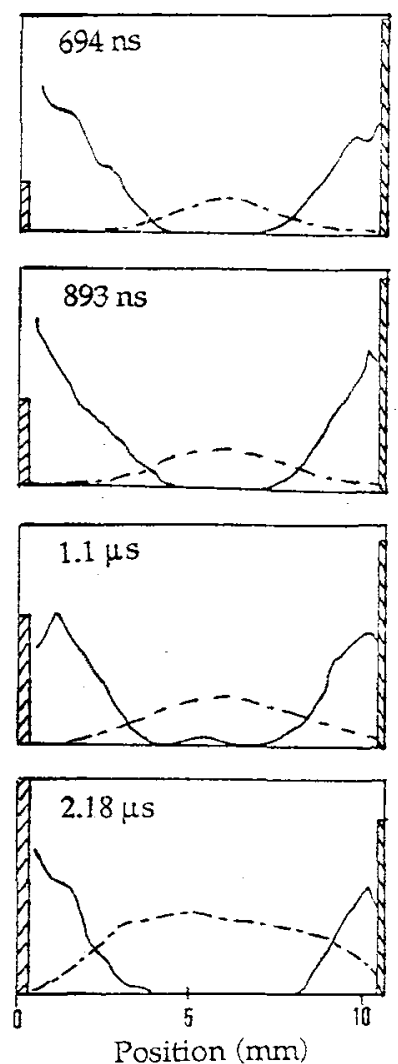

Fig 4:Space and time dependence of the Electric field (full line) and emission intensity $\mathrm{Ar}^{+} 457,9$ nm(dashed line) in the hollow cathode operated in pulsed conditions. The applied voltage and the current are represented by the height of the sticks. Same gas and discharge cell as Fig 3

\section{AKNOWLEDGMENTS.}

This work has been supported in part by DRET, contract 88-108.

\section{REFERENCES.}

1. E.A. Den Hartog, D.A. Doughty and J. Lawler, Phys. Rev. A38, 2471 (1988)

2. B.N. Ganguly, J.R. Shoemaker, B.L. Preppernau and A. Garscadden, J. Appl. Phys. 61, 2778 (1987)

3. B.N. Ganguly and A. Garscadden, Phys. Rev. A32, 2544 (1985)

4. T. Masaki, A. Wada, Y. Adachi, and C. Hirose, Appl. Spectr. 42, 49 (1988)

5. C. A. Moore, G.P. Davis and R.A. Gottscho, Phys. Rev. Lett. 52, 538 (1984);

6. R.A. Gottscho, G.R. Scheller, D. Stoneback and T. Intrator, J. Appl. Phys. 66, 492 (1989)

7. J. Derouard and N. Sadeghi, Opt. Comm. 57, 239 (1986);

8. H. Debontride, J. Derouard, P. Edel, R. Romestain, N. Sadeghi and J.P. Boeuf, Phys. Rev. A40, 5208 (1989)

9. M.P. Alberta and J. Derouard, J. Phys. D, Appl. Phys. 24, 904 (1991)

10. M.B. Radusky and A.J. Saykally, Chem. Phys. Lett. 152, 419 (1988)

11. Ph. Belenguer and J.P. Boeuf, Phys. Rev. A41, 4447 (1990)

12. K. Frank et al, IEEE Trans. Plasma Science, 17, 754 (1989)

13. J.P. Boeuf and L.C. Pitchford, IEEE Trans. Plasma Science, in press 